\title{
Secretion of Slime, the Extracellular Matrix of the Plasmodium, as Visualized with a Fluorescent Probe and Its Correlation with Locomotion on the Substratum
}

\author{
Hiromi Sesaki* and Satoshi Ogihara \\ Department of Biology, Graduate School of Science, Osaka University, Toyonaka, Osaka 560, Japan
}

Key words: Slime/extracellular matrix/Physarum/exocytosis/pseudopod formation/cell locomotion

\begin{abstract}
Slime, the extracellular matrix of Physarum plasmodium, is secreted by the exocytosis of a vesicles that contain a slime precursor. Using an antibody raised against biochemically purified slime, we detected the intracellular localization of the slime vesicle. Slime vesicles are abundant in the advancing front of the plasmodium, as confirmed by electron microscopic observation in two different cross-sectional angles. Screening various reagents, we found that rhodamine-phosphatidylethanolamine (Rh-PE) binds specifically to slime in both its intravesicular and extracellular forms, as confirmed by immunoelectron microscopy using an antibody against fluorochrome rhodamine. The plasmodia vitally stained with Rh-PE exhibited dynamic fluorescent patterns during the course of locomotion. The fluorescence was conspicuous at the periphery of the leading pseudopods and oscillated according to the shuttle streaming that accompanied the relaxation and contraction of the periphery; it was intense in the relaxation phase when pseudopods extended, and became weak in the contraction phase when pseudopods contracted. The results collectively mean that the slime vesicles carried by the cytoplasmic streaming accumulated prior to secretion at the advancing margin of the plasmodium.
\end{abstract}

Exocytotic vesicles play important roles in cell physiology of all eukaryotic cells. They release soluble contents to the extracellular space upon fusion with the plasma membrane. At the same time, they also supply new membrane materials to the plasma membrane including proteins and lipids. Macromolecules supplied in this way increase the surface area of the plasma membrane. Such an increase in the membrane components is accompanied by a variety of cell shape changes, such as pseudopod extension $(13,48)$, neurite elongation $(11,40)$, cytokinesis (10), and yeast budding (21). Exocytosis has been suggested to take place at the growth cone of growing neurons $(20,21)$, at the tips of extending buds of yeast (25), and at the cleavage furrows in dividing cells $(9,15)$. These localized exocytotic events implicate a role of exocytotic vesicles in cell shape changes as a supplier of new membrane materials to specific regions in cells, where dramatic expansion of the plasma membrane takes place.

In cells locomoting on substrate, the leading edge has been suggested to be a site for membrane insertion of exocytotic vesicles. In Hela cells, surface receptors for

\footnotetext{
* Present address: Charles H. Best Institute, University of Toronto, 112 College Street, Toronto, Ontario, Canada M5G 1L6.

Address for all correspondence: Satoshi Ogihara, Ph.D., Department of Biology, Graduate School of Science, Osaka University, Machikaneyama-cho, Toyonaka, Osaka 560, Japan.

Tel: +81-6-850-5811, Fax: +81-6-850-5817

e-mail: ogihara@bio.sci.osaka-u.ac.jp
}

transferrin and low density lipoprotein have been suggested to be inserted into the plasma membrane at the cell periphery based on their graded distribution with remarkable enrichment in the cell periphery $(12,14)$. In A431 cells, immuno-gold labeling revealed that the majority of the newly appearing transferrin receptors on the cell surface arise near the cell periphery (30). Since these receptors are transported to the cell surface by exocytotic mechanisms (27), these results suggest localized exocytosis occurring at the cell periphery. Furthermore, in locomoting $\mathrm{CHO}$ cells, immunofluorescence microscopy suggested that Golgi-derived vesicles are delivered to the plasma membrane at the leading edge carrying newly synthesized membrane glycoprotein, vascullar stomatis virus G-protein, which is used as a probe $(6,7$, 33). All these data strongly suggest the involvement of exocytosis in pseudopod formation. However, it has been difficult to directly correlate exocytosis to pseudopod formation, probably because the size of exocytotic vesicles is too small to be observed in live cells.

In Physarum plasmodia, we previously identified the vesicle which contains slime, the plasmodial extracellular matrix, and observed its exocytosis in psuedopodial regions using video-enhanced microscopy (45). Interestingly, a single exocytosis of the slime-containing vesicle was followed by extension of a single pseudopod. Quantitative analyses demonstrated a close correlation between the exocytotic events and the individual protrusion of pseudopods in terms of location, timing and 
size. These observations raise the question of whether the exocytosis of the slime-containing vesicles is also correlated to plasmodial locomotion, which results from accumulation of single pseudopod formation. In this paper, we visualized slime secretion in a live plasmodium by use of a specific fluorescent marker, rhodamineconjugated phosphatidylethanolamine, that binds to slime, and correlated the secretory activity of the slime vesicle to the overall locomotory activity of the plasmodium. When plasmodia were locomoting on substrate, slime appeared to be secreted at the leading edge, suggesting the correlation between exocytosis of the slimecontaining vesicle and plasmodial locomotion.

\section{MATERIALS AND METHODS}

Culture of Plasmodia. The plasmodium of Physarum polycephalum (Carolina Biological Supply Co., Burlington, USA) was fed with oatmeal and housed on damp towel paper set on glass petri dishes in a plastic case (16).

For observation, spread plasmodia were developed from the endoplasmic vein (5). In brief, the cytoplasm was isolated mechanically by drawing cytoplasm into a file glass microcapillary with internal diameter of $380 \mu \mathrm{m}$ at cut site, and extruding it into appropriate buffers. Isolated cytoplasm was then cut into $3.0 \mathrm{~mm}$ lengths with a razor blade under a binocular microscope. With electron microscopy, we confirmed that the plasma membrane resealed itself within $10 \mathrm{sec}$. As Baranowski and Wohlfarth-Bottermann (5) described, the cytoplasm began to spread like normal plasmodia, without any signficant time lag, when placed on a $1.5 \%$ agar substrate. It then took on a polarized shape having a leading edge and a tail, and exhibited directed movements at later times.

Electron Microscopy. Spread plasmodia were fixed in $2.5 \%$ glutaraldehyde, $1 \% \mathrm{OsO}_{4}$ and $50 \mathrm{mM} \mathrm{Na}$-cacodylate buffer at $\mathrm{pH} 7.0$ for $1 \mathrm{hr}$ at room temperature (45). They were washed three times in water, stained en bloc for $15 \mathrm{~min}$ in $2 \%$ uranyl acetate and carried through a series of dehydration steps in ethanol and propylene oxide. Specimens were embedded in a 50:50 Epon-araldite mixture. After ultrathin sectioning, sections were stained with uranyl acetate followed by lead citrate, and observed with a JEM-1200EX electron microscope at the acceleration voltage of $80 \mathrm{kV}$.

For morphometrical analysis, EM negatives were digitized with a high-resolution drum scanner (DT-S1030AI; Dainippon Screen Co., Kyoto, Japan) at the maximum resolution of $5,200 \mathrm{dpi}$. The digital images were stored on a $128 \mathrm{MB}$ magneto-optical disk with a Macintosh $660 \mathrm{AV}$ (Apple Computer, Cupertino, USA). The mean diameter of slime filaments was determined using NIH Image 1.44.

Slime Isolation and Anti-slime Antibody. Secreted slime was isolated by following the method previously described (46). In brief, $25 \mathrm{~g}$ of secreted slime left behind the tail of migrating plasmodia was collected, suspended in $20 \mathrm{mM}$ Tris$\mathrm{HCl}, \mathrm{pH} 7.6$, and homogenized by sonication for $5 \mathrm{~min}$. The slime was then washed by centrifugation at $6,000 \mathrm{rpm}$ for 10 min successively in three different solutions: first in the buffer containing $5 \mathrm{mM} \mathrm{CaCl}_{2}$, second in the buffer containing $67 \%$ ethanol and finally in water. Slime was resuspended in $1 \%$ cetylpyridium chloride and incubated finally with $0.8 \%$ cetylpyridinium chloride overnight at $37^{\circ} \mathrm{C}$ with rotation. The slime suspension was centrifuged at $6,000 \mathrm{rpm}$ for $10 \mathrm{~min}$ at $37^{\circ} \mathrm{C}$ and the resultant pellet was resuspended in $75 \mathrm{ml}$ of $2 \mathrm{M} \mathrm{NaCl}$ containing 10\% ethanol using Physcotron. After incubation in 3 volumes of ice cold ethanol for $1 \mathrm{hr}$, the slime was pelleted and washed twice in ethanol to remove salts. The slime was then dialyzed against water to a final protein concentration of $2 \mathrm{mg} / \mathrm{ml}$. Purified slime was injected into rabbits to obtain the polyclonal antibodies. Using a 200 -fold dilution of the antiserum yielded, the purified slime was detectable as low as $2 \mathrm{ng}$ in dot blot analysis.

Immunofluorescence Microscopy. Plasmodia were fixed in $3.7 \%$ formaldehyde containing $40 \mathrm{mM}$ phosphate buffer, $\mathrm{pH} \mathrm{7.0,} \mathrm{for} 30 \mathrm{~min}$ at room temperature. After washes in PBS, non-specific binding was blocked with PBS containing $1 \%$ BSA. Samples were incubated with anti-tetramethyl rhodamine antibody (Nordic Immunological Laboratories, Holland; 1:100 dilution in PBS containing 1\% BSA). After washes, samples were stained with FITC-conjugated anti-rabbit antibody (1:100 dilution) for $1 \mathrm{hr}$. Samples were washed and mounted in PBS containing 50\% glycerol and $0.5 \% \beta$ mercaptoethanol. Fluorescence images were acquired with a laser scanning microscope (LSM 200, Olympus, Tokyo, Japan).

Immunoelectron Microscopy. Plasmodia spread on the agar substrate were fixed in $1 \%$ glutaraldehyde, $4 \%$ formaldehyde, $0.2 \%$ picric acid, $0.5 \mathrm{mM} \mathrm{CaCl}_{2}$, and $100 \mathrm{mM}$ cacodylate- $\mathrm{NaOH}, \mathrm{pH} 7.0$, for $2 \mathrm{hr}$ at room temperature (8). Samples were incubated in $1 \mathrm{mg} / \mathrm{ml} \mathrm{NaBH}_{4}, 0.5 \mathrm{mM} \mathrm{CaCl}_{2}$, and 100 $\mathrm{mM}$ cacodylate- $\mathrm{NaOH}, \mathrm{pH} 7.0$, for $15 \mathrm{~min}$ on ice, and washed in $0.5 \mathrm{mM} \mathrm{CaCl}$, and $100 \mathrm{mM}$ cacodylate- $\mathrm{NaOH}$, $\mathrm{pH} 7.0$, for $20 \mathrm{~min}$, and $0.5 \mathrm{mM} \mathrm{CaCl}_{2}$ and $50 \mathrm{mM}$ malate$\mathrm{NaOH}, \mathrm{pH} 4.0$. Samples were post-fixed in $2 \%$ uranyl acetate containing $50 \mathrm{mM}$ malate- $\mathrm{NaOH}$ for 2 hours on ice. They were washed three times in water, stained en bloc for $15 \mathrm{~min}$ in $2 \%$ uranyl acetate and, carried though a series of dehydration steps in acetone. Specimens were embedded in LR Gold (Polyscience, Warrington, PA, USA). After ultrathin sectioning, sections were mounted on nickel grids and blocked with PBS containing 1\% BSA and $0.5 \%$ Tween-20, and stained with anti-rhodamine antibody (Nordic Immunological Laboratories, Holland; 1:100 dilution) followed by $20-\mathrm{nm}$ colloidal gold-conjugated anti-rabbit antibody (OD520 $=0.5$ ) (Zymed Laboratories, Inc., San Francisco, CA, USA). After washes, sections were stained with $2 \% \mathrm{OsO}_{4}$ for $15 \mathrm{~min}$ at room temperature.

Vital Staining of Plasmodia. EVs were stained in $0.5 \mathrm{mg}$ $/ \mathrm{ml}$ L-a-phosphatidylethanolamine-N-(lissamine rhodamine B sulphonyl) (Rh-PE) (Avanti Polar Lipids, Arabaster, AL, USA) in $10 \mathrm{mM}$ Tris-HCl, pH 7.6, for $30 \mathrm{~min}$ at room temper- 
ature and then washed three times in the same buffer without Rh-PE. One stained EV was incubated for 6-9 hours at $21^{\circ} \mathrm{C}$ together with one unstained EV on coverslips coated with $1.0 \%$ agar containing the buffer. As controls, plasmodia were stained with $0.05 \mathrm{mg} / \mathrm{ml} \mathrm{DiI}$ and $0.01 \%$ Eosin Y. These two EVs underwent fusion during incubation (17), and consequently there was often only one plasmodium spread on a coverslip. They were then observed under a confocal laser microscope (LSM-GB 100, Olympus, Tokyo, Japan). One fluorescence image was created by integration of 4 images taken at 1 sec intervals. We also used stained plasmodia without fusion and staining patterns of Rh-PE were indistinguishable from fused ones.

To quantitate the fluorescence intensity at the leading edge, we averaged the digitized fluorescence intensity of $100 \mu \mathrm{m}$ width along the direction of the pseudopod extension using NIH images. The peak intensity represented the fluorescence intensity of the leading edge. The cell margin was determined based on the fluorescent images, which was consistent with the images obtained by bright-field microscopy.

\section{RESULTS}

Certain vesicular structures accumulates at the frontal region of the plasmodium (Fig. 1). Those vesicles constitute a certain zone near the edge, as evident in the two kinds of electron micrograph sectioned parallel and perpendicular to the substrate (Fig. 1A and B). In this zone, the vesicles are loosely aggregated and touch each other while those in the rest of the cytoplasm behind the zone are distributed sporadically. The edge of the accumulation zone is often in contact with the actin meshwork immediately beneath the plasma membrane. The vesicle is $3.3 \pm 0.6 \mu \mathrm{m}(\mathrm{n}=187)$ in diameter on the average (45), and it is the largest organelle in the Physarum plasmodium except for a few lysosomes. The contents of the vesicle are conjectured to be slime, the extracellular matrix of the plasmodium $(45,49)$. Out of various granular structures, this vesicle is the only organelle that is found in the cortical actin meshwork (Fig. 1C).

Fig. 1. Ultrastructures of the Frontal Region of the Plasmodium. Spreading plasmodia were sectioned perpendicular (A) and parallel (B and $\mathrm{C}$ ) to the substrate. In $\mathrm{A}$, the lower side of the image corresponds to the substratum and the plasmodium is migrating toward the left side of the image. In B and C, the substratum is "below" the image, and the plasmodia are migrating upward in the images. Vesicular structure with a diameter of about $3-5 \mu \mathrm{m}$ are abundant at the very periphery of the pseudopodial region. As explained in the text, the vesicle contains slime, the extracellular matrix of the plasmodium of Physarum polycephalum. These vesicles are loosely aggregated in places to form an accumulation zone near the advancing front where lobopods of the plasmodium keep expanding. The slime vesicle is often found in the actin-rich cortical region. In $\mathrm{C}$, one slime vesicle actually touches the plasma membrane at the apical side. All slime vesicles were indicated with asterisks. Bar; $1 \mu \mathrm{m}$ in A, $5 \mu \mathrm{m}$ in B, and $3 \mu \mathrm{m}$ in C.
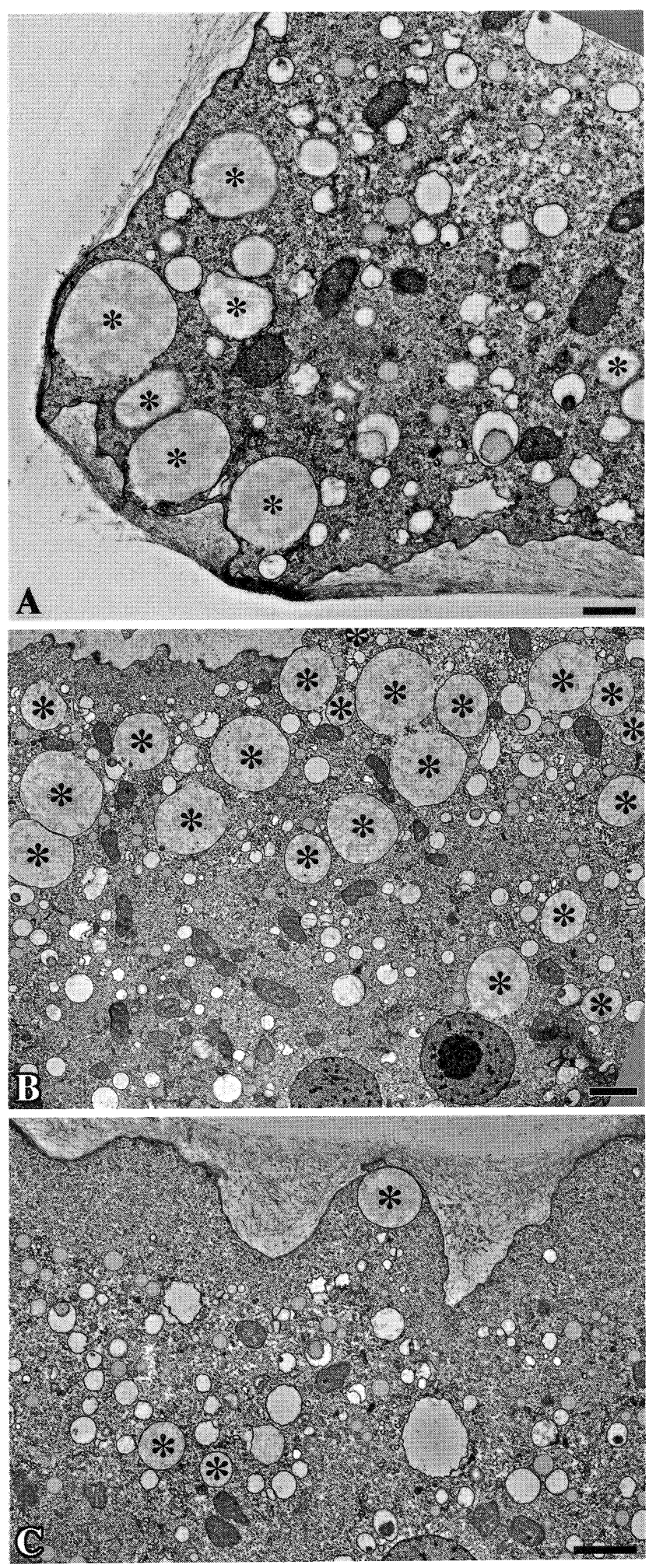

Fig. 1. 
We reported that the contents are delivered outside the plasmodia by an exocytotic process by analyzing the behavior of the vesicles in live plasmodium and by the electron microscopic observations of the exocytotic process (45). Slime secreted from such slime-containing vesicles forms a fibrous appearance on the plasmodium surface and thus covers the entire plasmodium surface. In this paper, we try to identify the contents of this vesicle to be slime in an alternative way as follows.

For the identification of the vesicle contents, we employed a biochemical method, combined with immunofluorescence. Following the isolation protocol of Shimada et al. (46), we isolated the slime and processed it for electron microscopy. Figure 2 compares the isolated slime with slime in the vesicles and the extracellular space. Slime fibrils before and after exocytosis are morphologically distinct. Those contained in the vesicles are electron-opaque, and become electron dense after exocytosis. The electron-dense nature of the isolated slime fibril resembles the exocytosed fibrils. Slime fibrils before and after exocytosis were similar in diameter $5.7 \pm 1.0 \mathrm{~nm}(\mathrm{n}=100)$ and $6.2 \pm 1.6 \mathrm{~nm}(\mathrm{n}=100)$, respectively. The isolated fibril had a diameter half the diameter of the fibrils in situ, i.e., $2.8 \pm 0.4 \mathrm{~nm}(\mathrm{n}=100)$.

Using the isolated slime as an antigen, we raised polyclonal antibodies in rabbits and stained the plasmodium by the immunofluorescence method. The plasmodium is generally thick, hence inappropriate for conventional fluorescent microscopy. For better resolution, we used the agar-overlay method $(26,32,39,45)$ and combined it with the optical sectioning method either by laser scanning microscopy or the digital deconvolution method with a cooled CCD camera (3).

Figures 3A-C are representative images of the antislime immunofluorescence. The structures identified by the antibodies are dots in the cytoplasm whose diameter is in the range of $2-10 \mu \mathrm{m}$; this matches well with the diameter of the slime-containing vesicle identified by electron microscopy (45). The dots are highly concentrated in the frontal regions of the plasmodium (Fig. 3A; lobopod), compared with other regions such as the strand parts which are less populated with dots (Figs. 3B-C). We observed some other signals much smaller than the large dots. There are some diffuse signals in the cytoplasm which are much more intense than the background level with the pre-immune serum (Fig. 3D), but the ultrastructural counterparts are not known. The slime layer is seen outside the plasmodium, depending on the location. As slime is virtually invisible with DIC optics, by these images we were able to demonstrate for the first time the overall morphology of slime layer as it extends throughout the plasmodium.

The specimen shown in Figs. 3E-G was prepared in a way different from those shown in Figs. 3A-C. The plasmodium was sandwiched between a PEI-coated cover-

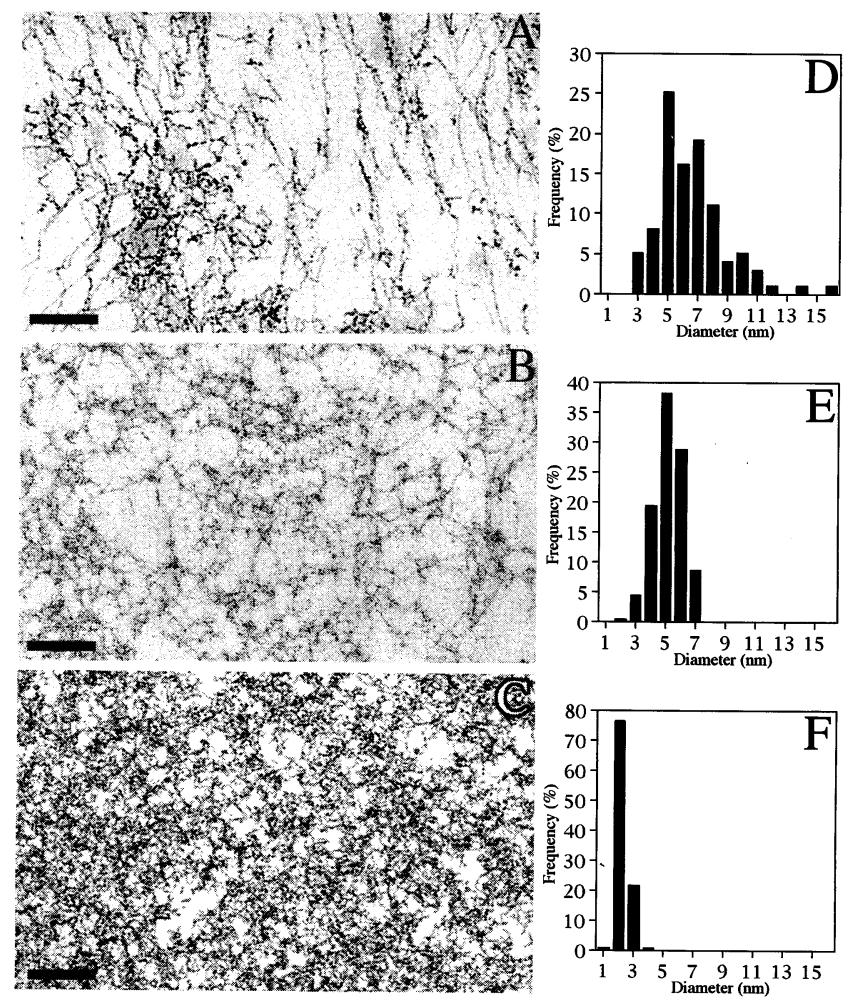

Fig. 2. Ultrastructures of Slime and the Diameter Distribution of the Fibrils. Three forms of slime fibrils, those secreted (A), those contained in the slime vesicle (B), and those purified biochemically (C), were fixed and processed for electron microscopy in the same way. The secreted slime is electron-dense while slime in the vesicle appears electron-opaque. Purified slime seems similar to secreted slime. The fibril size distribution shown in D, E, and F, which corresponds to A, B and $C$, respectively, show apparent shift in diameter to the thicker side, while the the average fibril diameter is $6.2 \mathrm{~nm}, 5.7 \mathrm{~nm}$ and 2.8 $\mathrm{nm}$, respectively. This may mean that the slime fibrils associate laterally on secretion. Bar; $200 \mathrm{~nm}$ in A and B, and $100 \mathrm{~nm}$ in C.

slip and a thin agar film (see Materials and Methods). In the thin intarval, the plasmodium was able to spread and migrate. Under these circumstances, however, the migration of the plasmodium is restricted to an almost two-dimensional space with very little freedom in the zaxis, and the secreted slime does not diffuse freely, but rather seems to stay where it is exocytosed. In this preparation, the hyaline layer has several protruding portions, and at the tip we see a very intense area of slime deposit. The slime layer is stained faintly but is not visible at all in the corresponding region with the DIC optics. These morphologies collectively suggest an exocytotic event of the slime vesicle and delivery of slime to the extracellular space by way of the hyaline layer. The protruding region thus becomes a part of the hyaline layer, implicating the mechanism of pseudopod extension (18).

Thus, we observed the accumulation of vesicles that 

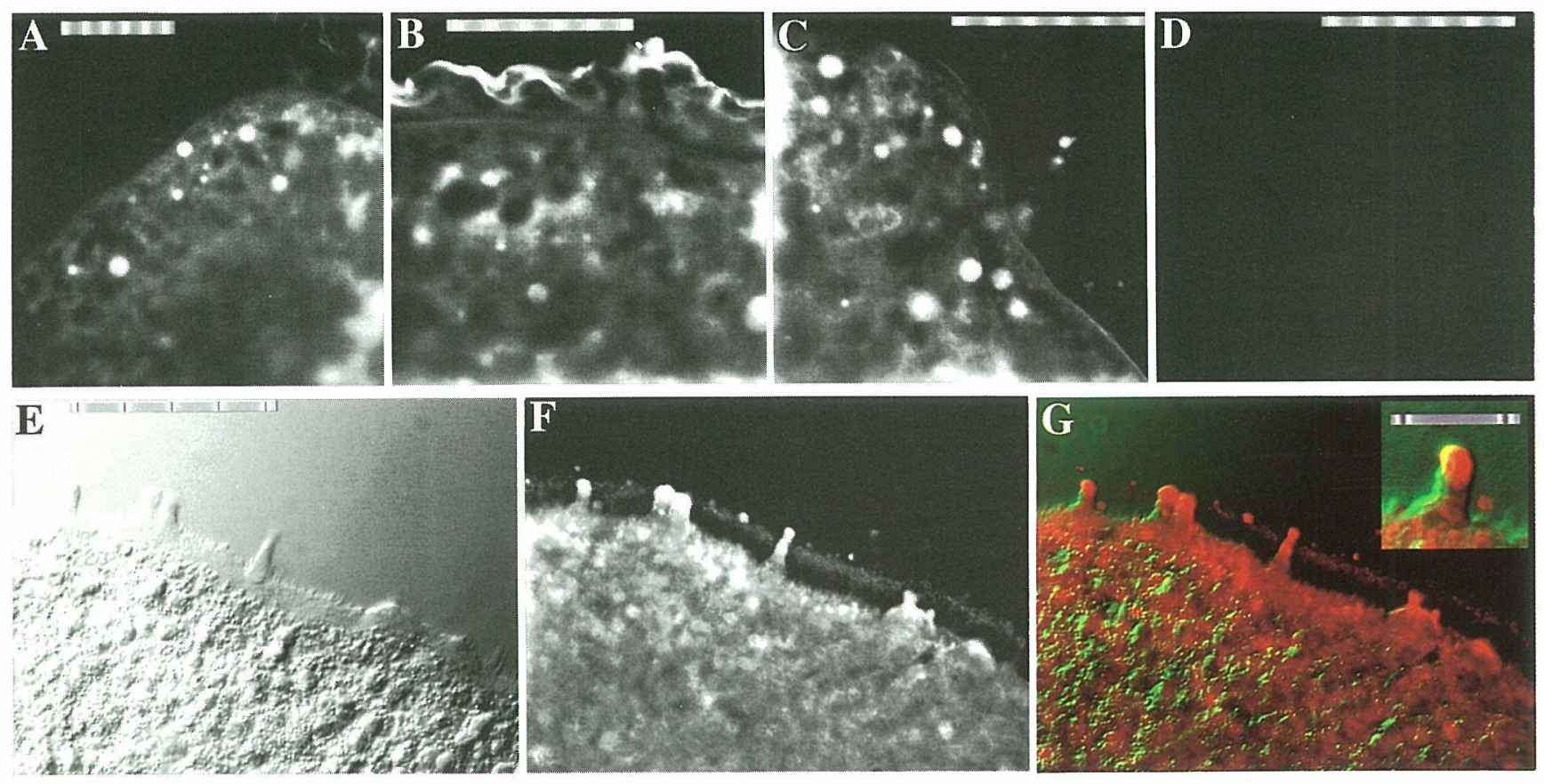

Fig. 3. Anti-slime Staining of the Plasmodium. Fluorescent images are all digitally deconvoluted, hence show optical sections. The antibodies raised against the purified slime stained the vesicular structures in the cytoplasm and the slime layer outside the plasmodium (A, B, and C). The diameter of fluorescent dots roughly corresponds to that of the slime vesicle shown in Fig. 1, although dots vary considerably in diameter and some dots exceeds $10 \mu \mathrm{m}$. The pre-immune control shows virtually no stain (D). The lower panel is a set of images of the same field: the DIC optics (E), immunofluorescence $(F)$ and the merged image with the DIC in green and the anti-slime signal in red $(\mathrm{G})$. Numerous dots are found in close proximity to the hyaline layer. Under conditions that restrict free diffusion of the materials exocytosed (see text for detail), the secreted slime is found at the tip of the protruded lobopods with the hyaline texture, indicating the coupling of slime secretion with the surface protrusion. Inset in $\mathrm{G}$ is a magnified view of one protrusion. Also note that the faint slime layer is covering the hyaline layer, although it is not visible in the DIC optics. Superimposed scales are all $10 \mu \mathrm{m}$ per unit.

contain slime at the leading edge of the plasmodium. Slime is likely to be secreted outward from the vesicles. We thought that the secretion of slime in this highly motile organism should be very vigorous, hence we made an attempt to visualize the slime secretion process in a live plasmodium so that its dynamic nature could be correlated with locomotion. After screening various reagents, we fortunately found that rhodamine-phosphatidylethanolamine (Rh-PE) stains the slime. DiI and eosin exhibited a specific staining pattern but did not correspond to slime distribution (data not shown). Rhodamine gave a stain quite close to Rh-PE, but was not used due to occasional unspecific staining patterns.

We first tried to confirm that Rh-PE binds to slime fibrils. To do this, we stained the live plasmodium with Rh-PE, and then fixed and processed it for immunostaining with the antibodies against rhodamine. Evidence is presented here at two levels of resolution, i.e., the immunofluorescence level and the immunoelectron microscopic level. The Rh-PE signal is found in the entire cytoplasm in optical microscopy. Image processing, however, reveals the signal consists of a cluster of dots (inset in Fig. 4A). The dot diameter is $3.55 \pm 1.22 \mu \mathrm{m}$ $(n=287)$ and is very close to the slime vesicle diameter

Fig. 4. Immunoelectron Microscopy with Anti-rhodamine. Live plasmodium was first stained with Rh-PE, and then fixed and processed for immunostaining. The three images in the left panel are the same field of the advancing front of the plasmodium: fluorescent image of Rh-PE (A), immunofluorescence of anti-rhodamine (B), and the merged image with the Rh-PE in red and the anti-rhodamine in green (C). While the Rh-PE signal is found throughout the cytoplasm, close examination with image processing reveals the stained regions are composed of dots. By contrast, the anti-rhodamine signal of the same field is predominantly not found in the cytoplasm but outside the plasmodium, except for the very tip of the front edge where the anti-rhodamine signal appears to be small patches and strands probably corresponding with surface invagination. The anti-rhodamine signal outside the plasmodium has punctuate appearances as shown in the inset of C. In D, E, and F, the anti-rhodamine signal was detected with colloidal gold particles at the electron microscopic level. The gold particles are predominantly found on the fibrous material outside the plasmodium (D). In a magnified view (E), it is obvious that the signal does not lie uniformly on the fibrils but has some regular spacing, which may correspond to the punctate staining pattern at the optical microscopic level. The gold particles are also found in slime vesicles (F). Bar; $100 \mu \mathrm{m}$ in A, $20 \mu \mathrm{m}$ in the inset of $\mathrm{A}, 1 \mu \mathrm{m}$ in D, $0.3 \mu \mathrm{m}$ in E, and $1 \mu \mathrm{m}$ in $\mathrm{F}$. The inset of $\mathrm{C}$ is the same magnification as the inset of $\mathrm{A}$. 

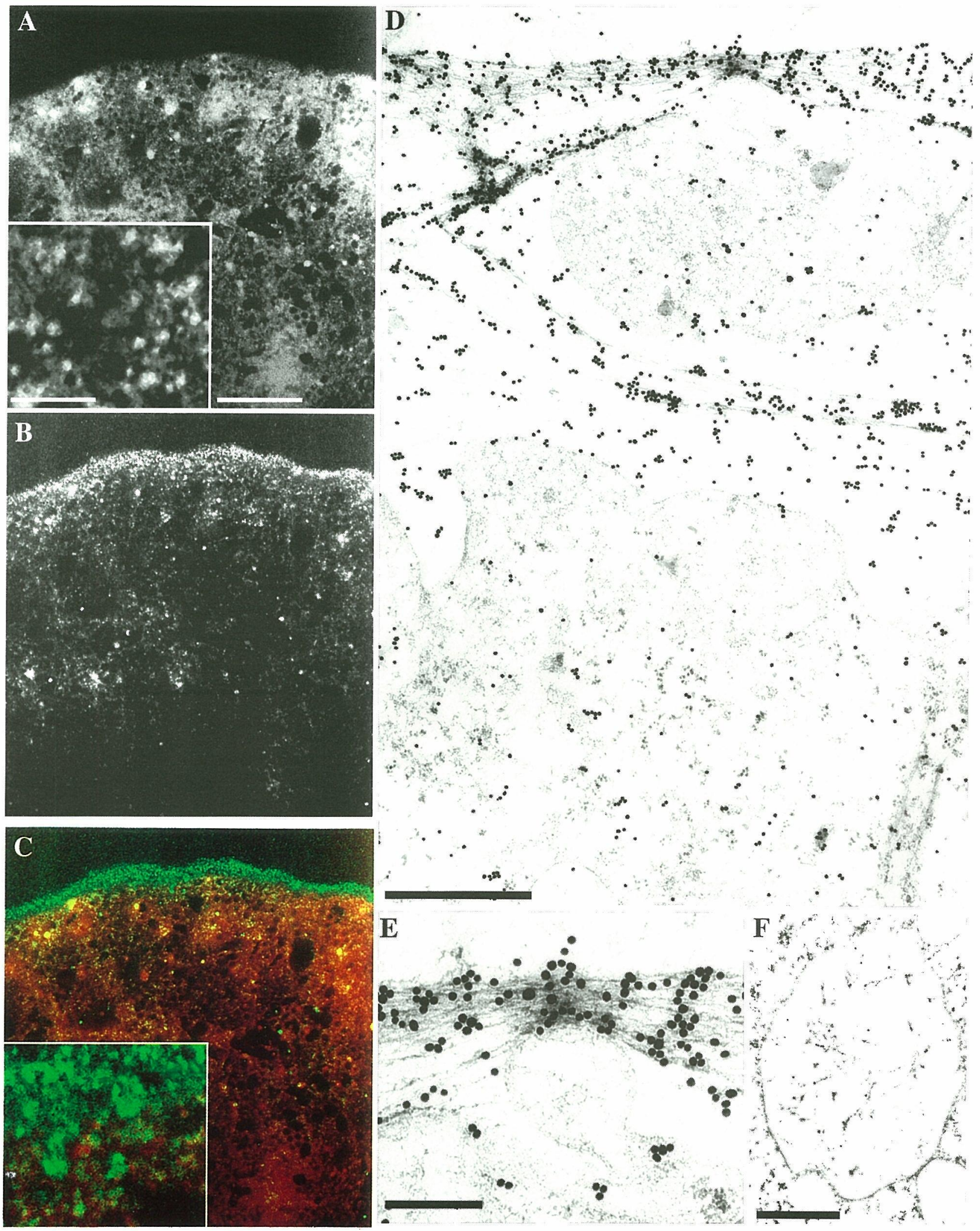

Fig. 4. 
obtained in electron microscopy (Fig. 1) and anti-slime immunofluorescence microscopy (Fig. 3). There are regions close to the edge of the advancing front with relatively strong fluorescence. Toward the inner portion of the cytoplasm, the fluorescence intensity gradually becomes lower. The presence of the yellow near the advancing front in the double stain image (Fig. 4C) suggests accumulation of the slime vesicles. As shown in the inset, the anti-rhodamine signal in green consists essentially of dots, either in the cytoplasm or in the extracellular milieu. The extracellular anti-rhodamine signal forms a belt covering the advancing front from outside. In marked contrast with the Rh-PE signal, the anti-rhodamine signal is quite low in the cytoplasm, but interestingly is very high in the extracellular space.

For immunoelectron microscopy, we employed the post-embedding method (8) to minimize the problem of accessibility of antibodies. The extreme abundance of the anti-rhodamine signal in the extracellular matrix rules out the possibility that the relatively low signal in the cytoplasm can be caused by the lowered penetration of the antibody (Fig. 4D). With the fixative that seems far less harsh to allow the post-embedding staining, the slime fibril is well preserved and the loss of cytoplasm is relatively suppressed. The gold particles are specifically present on the exocytosed slime fibrils outside the plasmodium. The slime fibrils seem to be bundled in places and the gold particles appear not to be distributed evenly on the slime fibrils but to have some sort of banding pattern. The contents of the slime vesicle are deposited with gold, although the signal is less abundant in vesicles than that in the extracellular matrix. The bulk cytoplasm virtually exhibits no specific staining of gold irrespective of the distance from the edge. In the control stain without the primary antibody, the number of gold particles was not significant (data not shown). By use of the two methods each with their distinct resolutions, it is obvious that both forms of slime, i.e., intracellular and extracellular, bind Rh-PE since both are identified with the anti-rhodamine antibody. A striking difference between the two forms of slime does not reside in the slime forms themselves, but in the bound Rh-PE. The
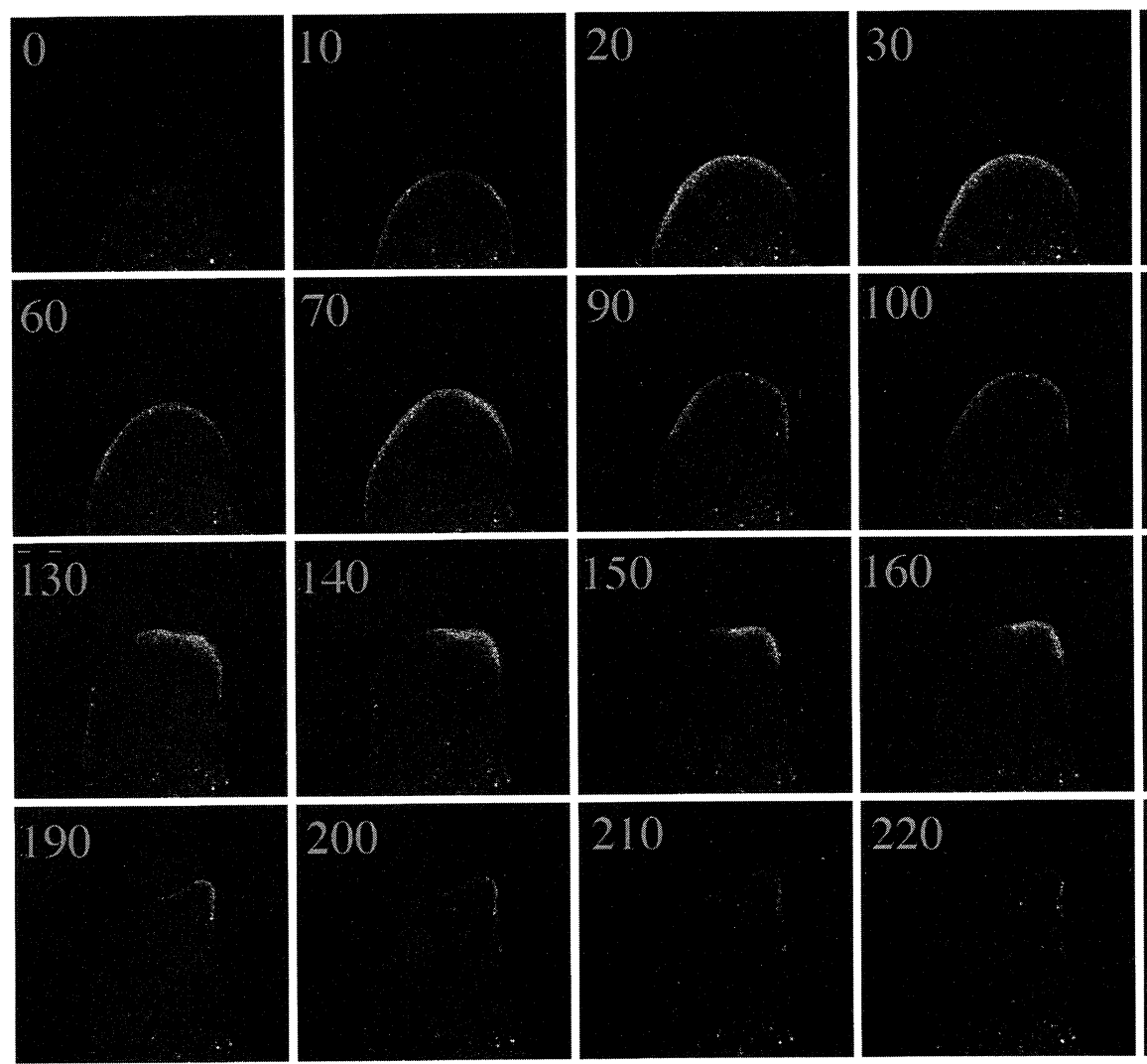
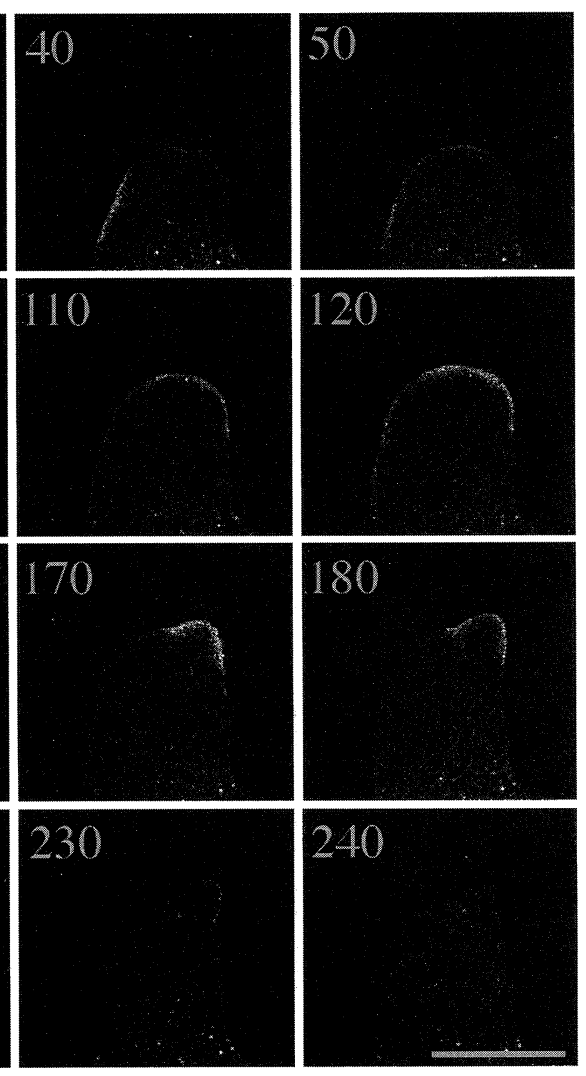

Fig. 5. Rh-PE Fluorescence Images in Live Plasmodium at the Lobopod. The plasmodium was vitally stained with Rh-PE and observed under a laser scanning microscope. The Rh-PE fluorescence signal is primarily detected at the edge of the advancing front of the plasmodium. The fluorescent intensity continues to increase and decrease while the lobopod keeps extending $\left(0^{\prime \prime}-190^{\prime \prime}\right)$. There is no fluorescence when there is no extension in the frontal regions of the plasmodium. When the lobopod ceases extension, the fluorescence disappears $\left(200^{\prime \prime}-240^{\prime \prime}\right)$. Time; sec. Bar; 0.5 $\mathrm{mm}$. 


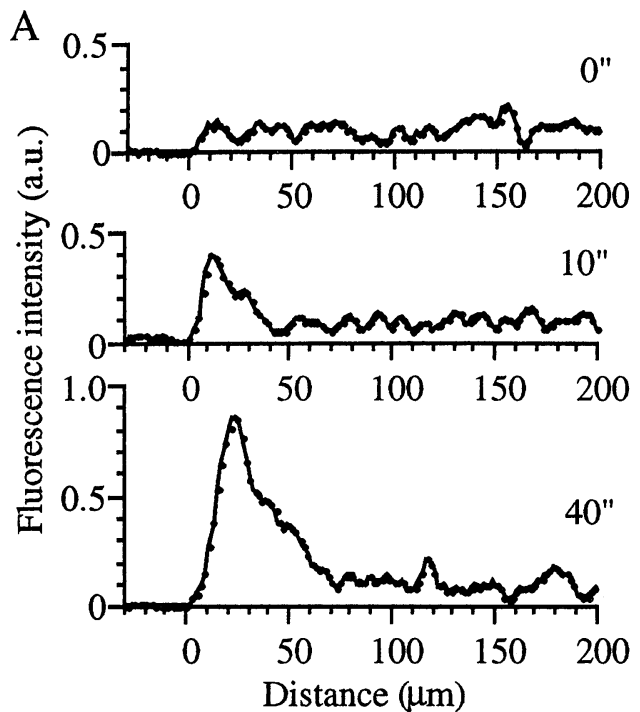

B
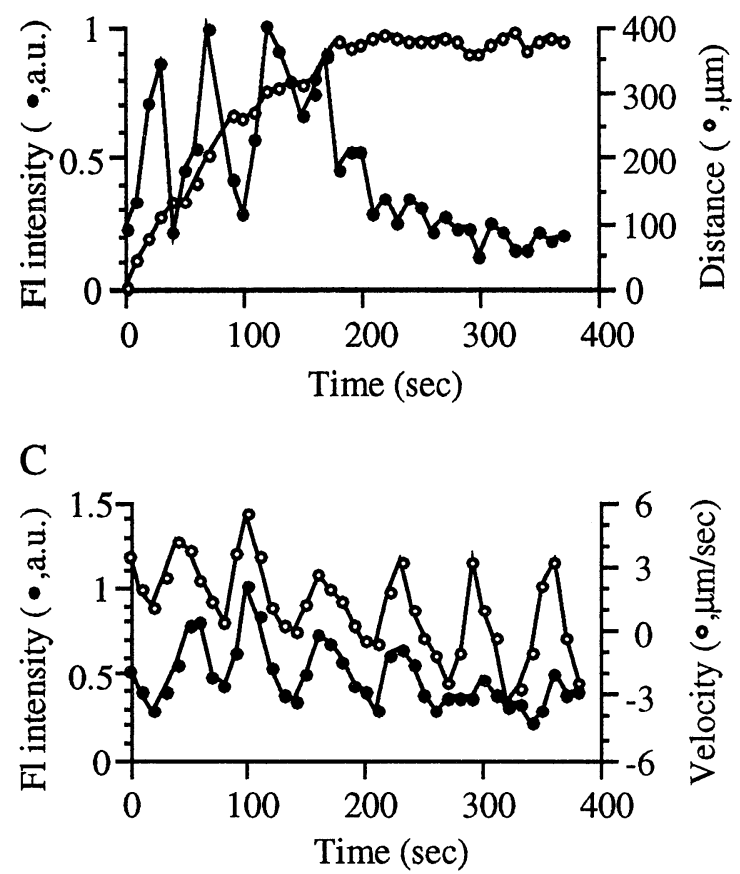

D

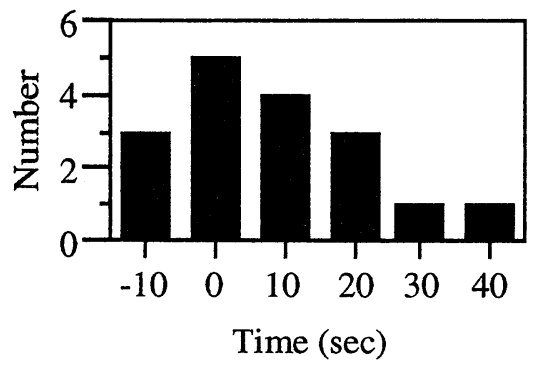

Fig. 6. fluorescence intensity of the Rh-PE is relatively high in the slime vesicles and becomes low when it is delivered outside the plasmodium by exocytosis of slime to which $\mathrm{Rh}-\mathrm{PE}$ is bound.

The direct observation of the secretion process of slime from the plasmodium was enabled as follows. To visualize a portion of the slime vesicles directly involved in the secretion event at the margin, an appropriate level of the Rh-PE fluorescence intensity had to be obtained. To do this, we used the cytoplasmic vein described previously (5). Cytoplasmic veins were prepared by puncturing the strand part of the plasmodium. Freshly gelled endoplasm was retrieved into a fine glass capillary and extruded into a buffer solution to make a fine endoplasmic vein (5). By doing so, most of the cytoplasmic surface is free of slime, hence Rh-PE is quite accessible to the cytoplasmic targets. This also minimized the possibility of the pre-existing extracellular slime to be stained by Rh-PE. The endoplasmic vein thus prepared was stained with Rh-PE as described above and allowed to spread on an agar film. They were observed with a laser scanning microscope with accelerated scanning rates.

The specific fluorescent pattern shown in Fig. 5 was observed in a restricted layer of the plasmodium at a distance of 50 to $200 \mu \mathrm{m}$ above the agar substrate. The RhPE fluorescence was dim and did not have any specific pattern in the other layers of the optical sections. Specific fluorescence was found at the rim of the cytoplasm of the advancing front to form an arc (Fig. 5). The fluorescence oscillated and the peak site of the fluorescence was stable at 10 to $20 \mu \mathrm{m}$ from the edge as shown in Fig. 6A. The fluorescence oscillation period is in the range of several tens of seconds and corresponds to the shuttle streaming period (31). We observed the flow of the endoplasm to and from the fluorescence site corresponding to the fluorescence oscillation. In contrast a consistent pattern was not observed in the plasmodium stained with DiI (data not shown).

The fluorescence oscillation was observable only

Fig. 6. Quantitative Analysis of Rh-PE Fluorescence Dynamics at the Leading Edge. (A) The Rh-PE fluorescence profile. Traces are those sampled at $0^{\prime \prime}, 10^{\prime \prime}$ and $40^{\prime \prime}$. The edge of the plasmodium is set at zero in $\mathrm{x}$-axis. The signal intensity at the peaks changes with time, but the fluorescence peaks are relatively stable in location and found 10$20 \mu \mathrm{m}$ inside of the pseudopod edge. (B) The relation of the fluorescence intensity to the distance of cell locomotion. When migration is vigorous, the fluorescence changes conspicuously. When migration stops, the fluorescence level decreases and its oscillation vanishes. (C) The fluorescence changes in parallel with the changes in the migration velocity. When the plasmodium edge expands, the fluorescence becomes intense. When the lobopods contract, the fluorescence decays. (D) The time difference between the peaks of fluorescence and the migration velocity. The time point at which the fluorescence intensity reaches the peak corresponds well with the time point at which the cell migration velocity reaches the maximum value. 
when the observed site showed locomotory activities. When migration ceased, the fluorescence disappeared and the oscillation became obscure. In the case shown in Fig. 5, a narrow portion of the advancing front became brighter (frames 130-180). The fluorescent portion continued to advance to make a protrusion in the region. As the fluorescence lowered, the deformation of the region ceased (frames 190-240). Thus the edge advancement was closely correlated with the changes in the fluorescence. The migration velocity was high when the fluorescence was high, and vice versa (Fig. 6C). The peak displacement between the velocity and the fluorescence was zero in some cases, and in other cases showed the fluorescence peak slightly behind the velocity peak (Fig. 6D).

\section{DISCUSSION}

The presence of a vesicular structure together with its relevance to the secretion of slime, the extracellular matrix of the Physarum plasmodium, was originally described by Stiemerling (49). It was first identified by electron microscopy and shown to be implicated in the secretion of slime by ruthenium red staining method (52). Recently, we identified the slime vesicle in live cells using high-resolution video-enhanced microscopy combined with electron microscopy of the same sample (45). In the present study, we further identified the slime vesicle by immunofluorescence and immunoelectron microscopy using the antibody raised against purified slime. The ultrastructure of purified slime is indistinguishable from the slime contained in the slime vesicle (Fig. 2). The antibody detects the vesicle contents as well as the slime on the extracellular layer (Fig. 3). Under conditions that suppress the diffusibility of the secreted slime, the slime was localized primarily at the tip of a protrusion from the lobopod with a hyaline texture (Figs. 3E$\mathrm{G})$. This is consistent with our previous observation that slime secretion leads to the surface protrusion of plasmodia at pseudopods (45). Although freshly secreted slime is virtually invisible with conventional optical microscopy, the obtained immunofluorescence pattern is consistent with the observation with electron microscopy (Fig. 1). Taken together, the present paper immunohistochemically demonstrates that the contents of the vesicle identified ultrastructurally by the WohlfarthBottermann group is slime. Slime is, therefore, secreted from the slime vesicle by an exocytotic process.

Slime secretion takes place at the leading edge of a pseudopod. Prior to secretion, the slime vesicles appear to be accumulated near the cell edge. The vesicular structures positive in the anti-slime fluorescence were quite crowded at the very margin of the frontal region of the plasmodium especially in the z-axis, so that even the deconvoluted images were sometimes blurred by comparison with the signals in the other thinly spread regions (Fig. 3F). Accumulation of the slime vesicles is also confirmed in electron microscopy. Ultrathin sections in two faces perpendicular to each other guarantees the accumulation of slime vesicles near the hyaline layer (Fig. 1). The Rh-PE staining of live cells also supports this view (Fig. 5).

Vesicle accumulation obviously requires a mechanism(s) by which only the slime vesicle is accumulated and invades the actin-rich cytoskeleton of the pseudopod. Although we have no direct evidence here, the interaction between exocytotic vesicles and the plasma membrane has been reported in other systems. Dense granules contained in platelets are docked on the inner side of the plasma membrane prior to secretion. Such anchorage of vesicles is supported by a specific structure ultrastructurally identified as bridges between the vesicle surface and the plasma membrane $(36,37)$. As to how slime vesicles invade the hyaline layer, probably the surface proteins bound to slime vesicle have properties of interacting with actin filaments cytoskeleton, either by severing the actin filaments comprising the hyaline pseudopod, or by sliding into the actin meshwork by motor proteins. Synaptic vesicles that proceed to the anchoring site at the presynapse through the dense actin meshwork is shown to have several actin binding proteins on the surface $(4,28,42)$.

Following the accumulation, the fusion of slime vesicles with the plasma membrane takes place at the leading edge of the pseudopod. Secretion of slime has been conjectured to take place in an exocytotic process by electron microscopic observations as follows $(45,49)$. First, only slime vesicles can invade the dense actin meshwork, approached from the granuloplasm toward the edge of the pseudopod. Some slime vesicles are closely positioned to the plasma membrane, and are often in contact with each other, thus forming an accumulation zone beneath the plasma membrane. Then, often in the neighboring regions, slimes vesicles are in contact with the plasma membrane (Fig. 1C). We did not see such a bridge-like structure between the two membranes as observed between the dense granules and the plasma membrane in platelets (37). Finally, the vesicle is fused with the plasma membrane and the contents are exposed to the extracellular space. The staining pattern shown in Fig. 3 strongly indicates exocytosis of slime; in the nearby region anti-slime positive dots are present in great abundance, suggesting the delivery of slime from the slime vesicles. This set of data adds an extra finding that slime section may be coupled with the protrusion of the pseudopod, as discussed below. Exocytosis of slime vesicle was observed in the live plasmodium using videoenhanced microscopy (45); slime vesicles first invade a pseudopodial region and then disappear near the advancing front of the plasmodium. Polarized secretion 
together with its biological function has been well documented not only in cell locomotion, but also in transport of proteins and lipids in MDCK cells (23), neurite extension $(20,21)$, cytokinesis $(9,15)$ and yeast budding $(22,25)$.

Slime secretion is coupled with surface contraction during cell locomotion. The fluorescence dynamics of $\mathrm{Rh}-\mathrm{PE}$ in the live plasmodium (Figs. 5 and 6) indicates that the rise in fluorescence may correspond with the accumulation of slime vesicles at the advancing front of the plasmodium. Accumulation may be followed by secretion, as supported also by the electron microscopic observations (Fig. 1) and the immunofluorescence observations (Fig. 3). If this is the case, then the fluorescence decay after the accumulation might be consistent with the finding that Rh-PE fluorescens more intensely when contained in slime vesicles than when it is exocytosed together with the slime. Presence of Rh-PE bound to secreted slime, together with its marked decrease in fluorescence intensity after secretion, is demonstrated by immunofluorescent and immunoelectron microscopy with antibodies against rhodamine (Fig. 4).

Our novel finding is that slime secretion and the cell's locomotory activity are closely correlated tightly with each other spatially and temporally, as revealed by the quantitative analysis of changes in Rh-PE staining pattern in live cells (Fig. 6). Prior to slime secretion, slime vesicles migrate toward the advancing front of the plasmodium. Slime is secreted through the ensuing accumulation step as explained above. Concomitant with these repeated cycles of accumulation and secretion, the bulk cytoplasm flows toward and back from the frontal region, in a rhythmic flow of cytoplasm in the plasmodium known as the shuttle streaming (31). Since the shuttle streaming is closely correlated with the contractionrelaxation cycle of the ectoplasm, the plasmodium cortex (38), we now know that the secretion of slime from the slime vesicles occurs in the contraction phase in the cortex, when the bulk cytoplasm flows back from the cortex. Also the accumulation of slime vesicles appears to take place in the relaxation phase. This notation is consistent with our observation shown in Fig. 5.

As to why Rh-PE fluorescence decreases after it is accumulated together with the bound slime at the cell edge, we believe one or more of the following possibilities to be true. First, a portion of the fluorescence decrease may reflect the portion of slime vesicles that flows back from the periphery, not being captured by the accumulation zone. Second, there may be lateral flow or even vertical flow of slime fibrils that would explain the decrease, especially when observed with a laser scanning microscope. Third, the fluorescence may be quenched in the aqueous environment, since the nonaqueous environment inside the slime vesicles is based on the hydration properties of the slime-type extracellu- lar matrix (Scott, 1989).

Acknowledgments. The authors would like to thank Masahiro Nakajima and Satoshi Tanaka of Osaka University for their help in image analyses. They are also grateful to Olympus Optical Co. Ltd. for use of a double channel laser scanning microscope, LSM200. This research was supported by a grant-in-aid (No. 06680685) from the Japanese Ministry of Education, Science and Culture.

\section{REFERENCES}

1. Achenbach, F., Achenbach, U., and WohlfarthBotTeRmANN, K.E. 1979. Plasmalemma invaginations, contraction and locomotion in normal and caffeine-treated protoplasmic drops of Physarum. Eur. J. Cell Bioil., 20: 12-23.

2. AChenbach, F. and Wohlfarth-Bottermann, K.E. 1986. Reactivation of cell-free models of endoplasmic drops from Physarum polycephalum after glycerol extraction at low ionic strength. Eur. J. Cell Biol., 40: 135-138.

3. Agard, D.A., Hiraoka, Y., Shaw, P., and Sedat, J.W. 1989. Fluorescence microscopy in three dimensions. Methods in Cell Biology, 30: 353-377.

4. Bahler, M., Benfenati, F., Valtorta, F., and Greengard, P. 1990. The synapsins and the regulation of synaptic function. Bioessays, 12: 259-263.

5. Baranowski, Z. and Wohlfarth-BottermanN, K.E. 1982. Endoplasmic veins from plasmodia of Physarum polycephalum: a new strand model defined age, structure, and behavior. Eur. J. Cell Biol., 27: 1-9.

6. Bergmann, J.E., Kupfer, A., and Singer, S.J. 1983. Membrane insertion at the leading edge of motile fibroblasts. Proc. Natl. Acad. Sci. USA, 80: 1367-1371.

7. Bergmann, J.E. and Singer, S.J. 1983. Immunoelectron microscopic studies of the intracellular transport of the membrane glycoprotein $(\mathrm{G})$ of vesicular stomatitis virus in infected Chinese hamster ovary cells. J. Cell Biol., 97: 1777-1781.

8. Berryman, M.A. and Rodewald, R.D. 1990. An enhanced method for post-embedding immunocytochemical staining which preserves cell membranes. J. Histochem. Cytochem., 38: 159-170.

9. Bluemink, J.G. and DelaAt, S.W. 1973. New membrane formation during cytokinesis in normal and cytochalasin-B-treated eggs of Xenopus laevis. I. Electron-microscopical observation. J. Cell Biol., 59: 89-108.

10. Bluemink, J.G. and DelaAt, S.W. 1977. Plasma membrane assembly as related to cell division. In The Synthesis, Assembly and Turnover of Cell Surface Components. (G. Poste and G.L. Nicolson, eds.). Elsevier/North Holland Biomedical Press, Amsterdam, pp.403-461.

11. BRAY, D. 1970. Surface movements during the growth of single explanted neurons. Proc. Natl. Acad. Sci. USA, 65: 905910.

12. BRETSCHER, M.S. 1983. Distribution of receptors for transferrin and low density lipoprotein on the surface of giant HeLa cells. Proc. Natl. Acad. Sci. USA, 80: 454-458.

13. BRETSCHER, M.S. 1984. Endocytosis: relation to capping and cell locomotion. Science, 224: 681-686.

14. BRETSCHER, M.S. and THOMSON, J.N. 1983. Distribution of ferritin receptors and coated pits on giant HeLa cells. EMBO J., 2: 599-603.

15. Byers, T.J. and Armstrong, P.B. 1986. Membrane protein redistribution during Xenopus first cleavage. J. Cell Biol., 102: 
2176-2184.

16. CAMP, W.G. 1936. A method of cultivating myxomycete plasmodia. Bull. Torrey Bot. Club., 63: 205-210.

17. Collins, O.R. and Haskins, E.F. 1970. Evidence for polygenic control of plasmodial fusion in Physarum polycephalum. $\mathrm{Na}$ ture, 226: 279-280.

18. CondeElis, J. 1993. Life at the leading edge: The formation of cell protrusions. Annu. Rev. Cell Biol., 9: 411-444.

19. Condeelis, J. and TAYloR, L.D. 1979. Cytoplasmic structure and contractility in amoeboid cells. In International Review of Cytology. vol.56 (G.H. Bourne, J.F. Danielli, eds.), Academic Press, New York, pp.57-144.

20. Craig, A.M., Wyborski, R.J., and Banker, G. 1995. Preferential addition of newly synthesized membrane protein at axonal growth cones. Nature, 375: 592-594.

21. DAI, J. and Sheetz, M.P. 1995. Axon membrane flows from the growth cone to the cell body. Cell, 83: 693-701.

22. Drubin, D.G. 1991. Development of cell polarity in budding yeast. Cell, 65: 1093-1096.

23. EAton, S. and Simons, K. 1995. Apical, basal, and lateral cues for epithelial polarization. Cell, 82: 5-8.

24. Edwards, C., Englert, D., Lotchaw, D., and Ye, H.Z. 1984. Light microscopic observation on the release of vesicles by isolated chromaffin cells. Cell Motil. Cytoskel., 4: 297-303.

25. Field, C. and ScheKman, R. 1980. Localized secretion of acid phosphatase reflects the pattern of cell surface growth in Saccharomyces cerevisiae. J. Cell Biol., 86: 123-128.

26. Fukui, Y., Yumura, S., and Yumura, K.T. 1987. Agar-overlay immunofluorescence: high resolution studies of cytoskeletal components and their changes during chemotaxis. Meth. in Cell Biol., 28: 347-356.

27. Goldstein, J.L., Brown, M.S., Anderson, R.G., Russell, D.W., and SchNeIDER, W.J. 1985. Receptor-mediated endocytosis: concepts emerging from the LDL receptor system. Annu. Rev. Cell Biol., 1: 1-39.

28. Greengard, P., Valtorta, F., Czernik, A.J., and Benfenati, F. 1993. Synaptic vesicle phosphoproteins and regulation of synaptic function. Science, 259: 780-785.

29. Henney, H.R., Jr. 1982. General Metabolism. In Cell Biology of Physarum and Didynium (H.C. Aldrich, and J.W. Daniel, eds.) Academic Press, London, pp.131-181.

30. HopkINS, C.R. 1985. The appearance and internalization of transferrin receptors at the margins of spreading human tumor cells. Cell., 40: 199-208.

31. Kamiya, N. 1959. Protoplasmic streaming. In Protoplasmatologia vol.8 (L.B. Heilbrunn and F. Weber, eds.) Springer, Wien, pp.1-199.

32. KAMIYA, N. and KurodA, K. 1965. Movement of the myxomycete plasmodium. I. A study of glycerinated models. Proc. Japan Acad., 42: 837-841.

33. Kupfer, A., Kronebusch, P.J., Rose, J.K., and Singer, S.J. 1987. A critical role for the polarization of membrane recycling in cell motility. Cell Motil. Cytoskel., 8: 182-189.

34. Lawson, D., RafF, M.C., Gomperts, B., Fertrell, C., and GilulA, N.B. 1977. Molecular events during membrane fusion: A study of exocytosis in rat peritoneal mast cells. J. Cell Biol., 72: 242-259.

35. MoolenaAR, W.H. 1995. Lysophosphatidic acid signaling. Cur. Opin. Cell Biol., 7: 203-210.
36. Morimoto, T., Ogihara, S., and Takisawa, H. 1990. Anchorage of secretion-competent dense granules on the plasma membrane of bovine platelets in the absence of secretory stimulation. J. Cell Biol., 111: 79-86.

37. Morimoto, T. and OGIhara, S. 1996. ATP is required in platelet serotonin exocytosis for protien phosphorylation and priming of secretory vesicles docked on the plasma membrane. $J$. Cell Sci., 109: 113-118.

38. Nagai, R., Yoshimoto, R.N., and KamiYA, N. 1978. Cyclic production of tension force in the plasmodial strand of Physarum polycephalum and its relation to microfilament morphology. J. Cell Sci., 33: 205-225.

39. Naib-Majani, W., Osborn, M., Weber, K., WohlfarthBottermann, K.E., Hinssen, H., and Stockem, W. 1983. Immunocytochemistry of the acellular slime mold Physarum polycephalum. I. Preparation, morphology, and reliability of results concerning cytoplasmic actomyosin patterns in sandwiched plasmodia. J. Cell Sci., 60: 13-28.

40. Popov, S., Brown, A., and Poo, M. 1993. Forward plasma membrane flow in growing nerve processes. Science, 259: 244246.

41. Rothman, J.E. 1994. Mechanisms of intracellular protein transport. Nature, 372: 55-63.

42. SCHWARz, T.L. 1994. Genetic analysis of neurotransmitter release at the synapse. Curr. Opin. Neurobiol., 4: 633-639.

43. ScotT, J.E. 1988. Secondary structures in hyaluronan solutions: chemical and biological implications. In The Biology of Hyaluronan (D. Evered and Whelan, eds.) John Wiley and Sons, New York, pp.6-15.

44. Segawa, A., Terakawa, S., Yamashina, S., and Hopkins, C.R. 1991. Exocytosis in living salivary glands: direct visualization by video-enhanced microscopy and confocal laser microscopy. Eur. J. Cell Biol., 54: 322-330.

45. SeSAKI, H. and OgIHARA, S. 1997. Protrusion of cell surface coupled with single exocytotic events of secretion of the slime in Physarum plasmodia. J. Cell Sci., In Press.

46. Shimada, Y., Murakami-Murofushi, K., Shioda, M., and OHTA, J. 1991. Extracellular polysaccharide from a true slime mold. Physarum polycephalum. Polymerization and autodegradation associated with cell growth, and its inhibitory activity on eukaryotic DNA polymerase $\alpha$. Natural Science Report, Ochanomizu University, 42: 39-47.

47. Shutt, D.C., Wessels, D., Wagenknecht, K., Chandrasekhar, A., Hitt, A.L., Luna, E.J., and Soll, D.R. 1995. Ponticulin plays a role in the positional stabilization of pseudopods. J. Cell Biol., 131: 1495-1506.

48. Singer, S.J. and KupfER, A. 1986. The directed migration of eukaryotic cells. Annu. Rev. Cell Biol., 2: 337-365.

49. Stiemerling, R. 1970. Production and secretion of slime of Physarum confertum. Cytobiologie, 1: 273-282.

50. Terakawa, S., Fan, J.H., Kumakura, K., and Imazumi, M. 1991. Quantitative analysis of exocytosis directly visualized in living chromaffin cells. Neurosci. Lett., 123: 82-86.

51. TImple, R. 1989. Structure and biological activity of basement membrane proteins. Eur. J. Biochem., 180: 487-502.

52. Wolf, K.V., Hoffmann, H.-U., and Stockem, W. 1981. Studies on microplasmodia of Physarum polycephalum: II. Fine structure and function of the mucus layer. Protoplasma, 107: 345-359. 